\title{
Extraction of 3D Shape from Optic Flow: a Geometric Approach
}

\author{
T.M.H. Dijkstra, P.R. Snoeren, C.C.A.M. Gielen \\ Lab. of Medical Physics and Biophysics, University of Nijmegen \\ P.O. Box 9101, $6500 \mathrm{HB}$, Nijmegen, The Netherlands \\ E-mail: tjeerd@mbfys.kun.nl, FAX +31 80541435
}

\begin{abstract}
We derive several measures of $3 D$ shape directly from the velocity field generated by a rigid curved surface patch under perspective projection. We use invariance under rotation of the image plane to decompose the velocity field in differential invariants. From a combination of these invariants we construct an approximation to the absolute value of the shape index, to the curvedness and to the principal direction. We show the effect of these approximations to be small for a field of view of 6 deg.

Keywords: $3 D$ shape, structure from motion
\end{abstract}

\section{Introduction}

Central in our approach to the extraction of structure from motion is the use of geometry. We study the invariance of the velocity field under the group of rotations of the plane (the Lie group SO(2)). The differential geometric properties of smooth surfaces can be classified according to order. The zeroth order property is the distance of the object. First order properties can be described by the slant and the tilt. Note that distance, slant and tilt of an object will in general change when it moves relative to an observer. The second order properties can be described by e.g. the principal curvatures and the principal direction. The principal curvatures are the lowest order properties which do not change when the object moves relative to the observer i.e. they are intrinsic to the object.

Because the velocity field is derived from a projection metric information cannot be obtained from it. This means that one cannot obtain the 3D translation velocity of the observer relative to the object. Neither can one obtain the distance to the object nor its curvatures. Of course one can construct combinations of these properties that do not depend on metric information e.g. the ratio of the principal curvatures. Since the principal curvatures are the lowest order properties that are intrinsic, the ratio of the principal cur- vatures (or any function thereof) is the only intrinsic property of the object that can be derived from the velocity field (up to second order).

\section{Description of shape measures}

In computer vision shape is often described by the principal curvatures $\kappa_{\max }$ and $\kappa_{\min }$ and the principal direction $\alpha_{0}$. The principal curvatures are the extrema of the normal curvature $\kappa_{n}$. The normal curvature is obtained as the curvature of the curve one gets when one cuts the surface with a plane through the normal of the surface. Koenderink and van Doorn introduced the following descriptors [6]:

$$
\begin{aligned}
S & =2 / \pi \arctan \left(\frac{\kappa_{\max }+\kappa_{\min }}{\kappa_{\max }-\kappa_{\min }}\right) \\
C & =\sqrt{\left(\kappa_{\max }^{2}+\kappa_{\min }^{2}\right) / 2}
\end{aligned}
$$

with the shape index $S$ carrying all scale independent information about shape, and the curvedness $C$ carrying all scale dependent information. Algebraically, one can view the shape index and the curvedness as scaled polar coordinates in the $\kappa_{\max }, \kappa_{\min }$ half-plane (see fig. 1). We take $\kappa_{\max } \geq \kappa_{\min }$ because interchanging $\kappa_{\max }$ and $\kappa_{\min }$ has the same effect as interchanging $x$ and $y$ and thus only changes the orientation of the surface, not its shape.

We parametrize a smooth surface patch using the range function $Z(X, Y)$ with $(X, Y, Z) \in \mathbf{R}^{3}$. We develop the patch up to second order in a Taylor series:

$$
\begin{aligned}
Z(X, Y)= & Z_{0}+Z_{X} X+Z_{Y} Y+ \\
& 1 / 2 Z_{X X} X^{2}+Z_{X Y} X Y+1 / 2 Z_{Y Y} Y^{2},
\end{aligned}
$$

where $Z_{0}$ is the distance to the patch, $\left(Z_{X}, Z_{Y}\right)^{T} \equiv$ $\nabla Z$ is the range gradient and $Z_{X X}, Z_{X Y}$ and $Z_{Y Y}$ denote the second order derivatives. The range gradient is related to the attitude of the surface by $\nabla Z=\tan \sigma(\cos \tau, \sin \tau)^{T}$ with $\sigma \in[0, \pi / 2]$ denoting the slant and $\tau \in[0,2 \pi)$ denoting the tilt. The slant 


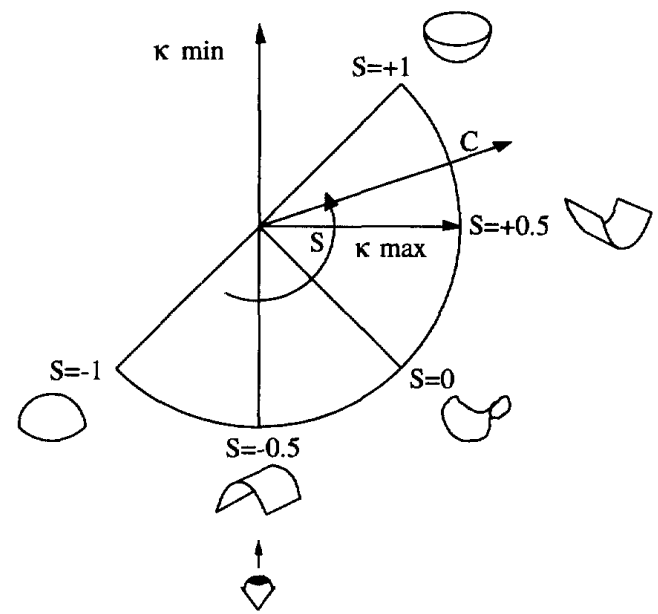

Figure 1 Objects in $\kappa_{\max }, \kappa_{\min }$ space. By a change of coordinates, objects can be characterized by an angular coordinate, the shape index $S$, and a radial coordinate, the curvedness $C$. The shapes drawn to illustrate various values of $S$ are to be viewed from below.

equals the angle between the normal of the surface and the line of sight (we take the direction of the normal always towards the viewer). The tilt equals the angle between the normal of the surface when projected on the image plane and the $X$-axis.

The relation between normal curvature and the second order derivatives of the range function depends also on the first order derivatives. Taking a direction $\mathbf{e}_{\alpha}$ in the tangent plane (which has angle $\alpha$ with the $X$-axis) and expressing the first order derivatives in terms of slant and tilt, we have $[7, \mathrm{p} 221]$;

$$
\begin{aligned}
& \lambda(\alpha) \equiv \kappa_{n}(\alpha) \frac{1+\tan ^{2} \sigma \cos ^{2}(\alpha-\tau)}{\cos \sigma}= \\
& Z_{X X} \cos ^{2} \alpha+2 Z_{X Y} \cos \alpha \sin \alpha+Z_{Y Y} \sin ^{2} \alpha,
\end{aligned}
$$

where we introduced $\lambda$ as a new curvature measure. In contrast to $\kappa_{n}$ which only depends on curvature, $\lambda$ also depends on the attitude of the tangent plane. Later we show that it is impossible to obtain $\kappa_{n}$ directly from the velocity field, but that $\lambda$ can be obtained directly. One can show that for:

$$
\tan \left(2 \alpha_{0}\right)=2 Z_{X Y} /\left(Z_{X X}-Z_{Y Y}\right),
$$

one obtains the maximal and minimal $\lambda$, given by:

$$
\begin{aligned}
& \lambda_{(\max , \min )}=1 / 2\left(Z_{X X}+Z_{Y Y}\right) \pm \\
& \quad 1 / 2 \sqrt{Z_{X X}^{2}+4 Z_{X Y}^{2}+Z_{Y Y}^{2}-2 Z_{X X} Z_{Y Y}} .
\end{aligned}
$$

When the tangent plane is fronto-parallel, we have $\sigma=0$ and $\lambda_{\max }, \lambda_{\min }$ are equal to $\kappa_{\max }, \kappa_{\min }$. Also, when the slant is not too large, $\tan \sigma$ will be close to 0 and $\cos \sigma$ will be close to 1 and thus $\lambda_{\max }, \lambda_{\min }$ will not deviate much from $\kappa_{\max }, \kappa_{\min }$.

\section{Invariants of the velocity field}

The decomposition of the zeroth and first order velocity field leads to four invariants [3]: translation of order zero and divergence $(\nabla \cdot \mathbf{v})$, rotation $(\nabla \times \mathbf{v})$ and deformation $(\nabla \circ v)$ of order one. In fig. 2 we show examples of the vector field generated by these invariants. Discussing these will give us a chance to introduce the weight of an invariant [2]. The weight captures the symmetry of the invariant under rotation: $2 \pi$ divided by the weight is the smallest angle over which one has to rotate the invariant so that it equals itself (except for weight zero). The translation has weight 1 , meaning that a rotation over $2 \pi$ maps the vector field onto itself. The divergence and rotation have weight 0 , meaning that every rotation maps the vector field onto itself. Thus they are scalars. The deformation has weight 2 , meaning that a rotation over $\pi$ maps the vector field onto itself.

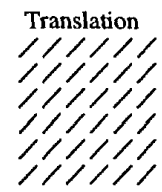

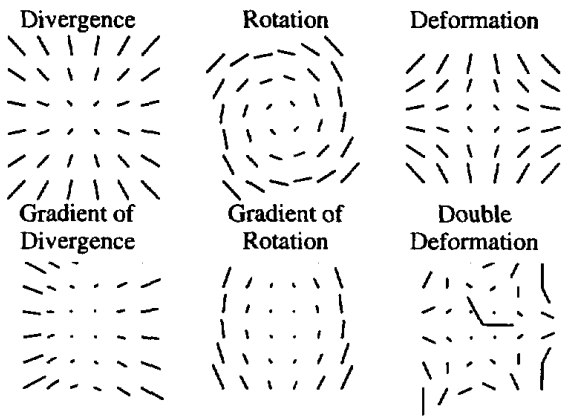

Figure 2 Flow fields generated by differential invariants. Top: zeroth order invariant in direction $(1,1)$. Middle: first order invariants, all in direction $(1,0)$. Bottom: second order invariants, all in direction $(1,0)$. To see the symmetry of the double deformation we have drawn two lines of equal length with an angle $2 \pi / 3$ between them in the plot of its flow field.

The image plane is two dimensional and has the nice property that rotations commute i.e. the result of two rotations is independent of the order in which they are performed. Because of this all invariants are 
complex numbers [2], with the exception of the invariants of weight 0 , which form a pair of real numbers. Because the group of rotations in the plane ( $\mathrm{SO}(2))$ is also easily denoted by a complex number of unit length we will construct the invariants using a complex number notation. We denote the velocity field by $v(z, \bar{z})$ with $z=x+i y$ and the complex conjugate $\bar{z}=x-i y . \quad v$ is now a mapping from the complex plane to the complex plane. We write the second order development of the velocity field as:

$$
v(z, \bar{z})=1 / 2 v_{z z} z^{2}+v_{z \bar{z}} z \bar{z}+1 / 2 v_{\bar{z} \bar{z}} \bar{z}^{2},
$$

with $v_{z z}$ the velocity field differentiated twice with respect to $z$ and with similar notation for the other derivatives. Now $v_{z z}, v_{z \bar{z}}$ and $v_{\bar{z} \bar{z}}$ are observables of our velocity field relative to some coordinate system. To make these observables independent of the coordinate system we rotate it over an arbitrary angle. It turns out that $v_{z z}, v_{z \bar{z}}$ and $v_{\bar{z} \bar{z}}$ are already invariant! This means that we cheated by starting with a very favourable representation from the outset. Rotating $v(z, \bar{z})$ over $n \theta$ we find:

$$
\begin{aligned}
v(z, \bar{z})= & 1 / 2 \exp (-i n \theta) v_{z z} z^{2}+\exp (i n \theta) v_{z \bar{z}} z \bar{z}+ \\
& 1 / 2 \exp (3 i n \theta) v_{\bar{z} \bar{z}} \bar{z}^{2}
\end{aligned}
$$

Thus we have three invariants of second order: $v_{z z}$ of weight $-1, v_{z \bar{z}}$ of weight 1 and $v_{\bar{z} \bar{z}}$ of weight 3 . The sign of the weight has to do with symmetry under reflection in the $x$ axis and need not concern us. One can change the sign by complex conjugation. The invariants are not unique: linear combinations of invariants with the same absolute weight are also invariant.

Rewriting the invariants in real coordinates we get:

$$
\begin{aligned}
& 4 v_{z z}=\left(\begin{array}{c}
2 v_{x y}^{y}+v_{x x}^{x}-v_{y y}^{x} \\
2 v_{x y}^{x}-v_{x x}^{y}+v_{y y}^{y}
\end{array}\right) \equiv \boldsymbol{\alpha}, \\
& 4 v_{z \bar{z}}=\left(\begin{array}{c}
v_{x x}^{x}+v_{y y}^{x} \\
v_{x x}^{y}+v_{y y}^{y}
\end{array}\right) \boxminus \boldsymbol{\beta}, \\
& 4 v_{\bar{z} \bar{z}}=\left(\begin{array}{c}
v_{x x}^{x}-v_{y y}^{x}-2 v_{x y}^{y} \\
v_{x x}^{y}-v_{y y}^{y}+2 v_{x y}^{x}
\end{array}\right) \equiv \gamma,
\end{aligned}
$$

with e.g. $v_{y y}^{x}$ denoting the second order derivative in the $y$ direction of the $x$ component of the velocity field. Here we introduced the second order invariants, denoted by $\boldsymbol{\alpha}, \boldsymbol{\beta}$ and $\boldsymbol{\gamma}$. Alternatively, we can express the invariants in nabla notation. We obtain the gradient of divergence $\nabla(\nabla \cdot \mathbf{v})=2\left(\bar{v}_{z z}+v_{z \bar{z}}\right)$ and the gradient of rotation $\nabla(\nabla \times \mathbf{v})=2 i\left(\bar{v}_{z z}-v_{z \bar{z}}\right)$ which were introduced in [3]. The double deformation $\nabla \circ \nabla \circ \mathbf{v}=4 v_{\bar{z} \bar{z}}$ is the remaining invariant. In fig. 2 bottom row we have plotted examples of the vector fields of these invariants.

\section{Second order invariants and shape}

We consider a moving observer viewing a stationary object. We locate the origin of our coordinate system at the vertex of perspective projection and the positive $Z$-axis along the line of sight. Subjecting the observer to a translation $\mathbf{V}=\left(V^{X}, V^{Y}, V^{Z}\right)^{T}$ and rotation $\Omega=\left(\Omega^{X}, \Omega^{Y}, \Omega^{Z}\right)^{T}$ we find for the velocity field on a planar camera at unit focal distance from the point of projection [8]:

$$
\begin{aligned}
\left(\begin{array}{c}
v^{x} \\
v^{y}
\end{array}\right)= & \left(\begin{array}{l}
V^{Z} / Z x-V^{X} / Z \\
V^{Z} / Z y-V^{Y} / Z
\end{array}\right)+ \\
& \left(\begin{array}{l}
\Omega^{X} x y-\Omega^{Y}\left(1+x^{2}\right)+\Omega^{Z} y \\
\Omega^{X}\left(1+y^{2}\right)-\Omega^{Y} x y-\Omega^{Z} x
\end{array}\right),
\end{aligned}
$$

with $x=X / Z, y=Y / Z$. Using eq. 3 we approximate $1 / Z$ in camera coordinates:

$$
\begin{aligned}
& 1 / Z(x, y)=\left(1 / Z_{0}\right)\left(1-Z_{X} x-Z_{Y} y\right)- \\
& 1 / 2 Z_{X X} x^{2}-Z_{X Y} x y-1 / 2 Z_{Y Y} y^{2}+C^{3}(x, y) .
\end{aligned}
$$

Substituting this in eq. 9 and then using eqs 6 to 8 , we get for the second order differential invariants:

$$
\begin{aligned}
& \alpha=\left(\begin{array}{cc}
Z_{X X}-Z_{Y Y} & 2 Z_{X Y} \\
2 Z_{X Y} & -Z_{X X}+Z_{Y Y}
\end{array}\right) \mathbf{v}_{\|}+ \\
& 4 J \Omega_{\|}-4 V_{\perp} / Z_{0} \nabla Z \text {, } \\
& \boldsymbol{\beta}=\left(Z_{X X}+Z_{Y Y}\right) \mathbf{V}_{\|}+2 J \boldsymbol{\Omega}_{\|}-2 V_{\perp} / Z_{0} \nabla Z \text {, } \\
& \gamma=\left(\begin{array}{cc}
Z_{X X}-Z_{Y Y} & -2 Z_{X Y} \\
2 Z_{X Y} & Z_{X X}-Z_{Y Y}
\end{array}\right) \mathbf{v}_{\|},
\end{aligned}
$$

where we have used the notation $\mathbf{V}_{\|} \equiv\left(V^{X}, V^{Y}\right)^{T}$ for translation parallel to the camera and $V_{\perp}$ for translation orthogonal to the camera and identical notation for $\boldsymbol{\Omega}$. The matrix $J$ denotes a rotation over $\pi / 2$.

To see its geometrical meaning we will calculate the length of $\gamma$ and the angle between $\gamma$ and $\mathbf{V}_{\|}$:

$$
\begin{aligned}
|\gamma| & =\left|\lambda_{\max }-\lambda_{\min }\right|\left|\mathbf{V}_{\|}\right|, \\
L\left(\mathbf{V}_{\|}, \gamma\right) & =-2 \alpha_{0},
\end{aligned}
$$

where we used eqs. 4 and 5 . Geometrically, $\gamma$ can be viewed as a rotation over $-2 \alpha_{0}$ followed by a scaling with $\lambda_{\max }-\lambda_{\min }$.

Neglecting the non-curvature dependent terms in $\beta$, which is equivalent to parallel projection [1]:

$$
\boldsymbol{\beta}=\left(\lambda_{\max }+\lambda_{\min }\right) \mathbf{V}_{\|} \text {. }
$$

Geometrically, $\boldsymbol{\beta}$ can be viewed as a scaling with $\lambda_{\max }+\lambda_{\min }$. Neglecting the non-curvature dependent terms in $\alpha$ :

$$
\begin{aligned}
|\boldsymbol{\alpha}| & =\left|\lambda_{\max }-\lambda_{\min }\right|\left|\mathbf{V}_{\|}\right|, \\
\angle\left(\mathbf{V}_{\|}, \boldsymbol{\alpha}\right) & =2 \alpha_{0} .
\end{aligned}
$$


Geometrically, $\alpha$ can be viewed as a rotation over $2 \alpha_{0}$ followed by a scaling with $\lambda_{\max }-\lambda_{\min }$.

Assuming that $\lambda_{\max }, \lambda_{\min }$ are close to $\kappa_{\max }, \kappa_{\min }$, we obtain the shape index using eq. 1 :

$$
S_{e}=\operatorname{sign}\left(\boldsymbol{\beta} \cdot \mathbf{V}_{\|}\right) 2 / \pi \arctan \left(\frac{|\boldsymbol{\beta}|}{|\boldsymbol{\gamma}|}\right) .
$$

The sign of the shape index can be obtained from the relation between $\boldsymbol{\beta}$ and $\mathbf{V}_{\|}$. Because $\boldsymbol{\beta}$ is parallel to $\mathbf{V}_{\|}$, we give $S_{e}$ a positive sign when $\boldsymbol{\beta}$ and $\mathbf{V}_{\|}$point in the same direction and we give $S_{e}$ a negative sign when the angle between $\boldsymbol{\beta}$ and $\mathbf{V}_{\|}$is $\pi$. So, all we need is an estimate of the direction of $\mathbf{V}_{\|}$. Unfortunately, this cannot be obtained from second order optic flow when we neglect the non-curvature dependent terms [1]. Here we assume that we obtained the direction of $\mathbf{V}_{\|}$by other means e.g. from knowledge of movement direction in active vision or from a third view [4].

Because $\boldsymbol{\beta}$ and $\mathbf{V}_{\|}$are parallel, we obtain an estimate of the principal direction $\alpha_{0}$ by:

$$
2 \alpha_{e}=\angle\left(\boldsymbol{\gamma}, \operatorname{sign}\left(\boldsymbol{\beta} \cdot \mathbf{V}_{\|}\right) \boldsymbol{\beta}\right)
$$

Thus, we can also find the principal direction directly from the velocity field. Note that when we do not know the direction of $V_{\|}$then we do not know which orientation is the direction of maximal curvature and which of minimal curvature. An estimate for the velocity scaled curvedness, $\Gamma_{e}$, can be obtained from:

$$
\Gamma_{e} \equiv C_{e}\left|\mathbf{V}_{\|}\right|=1 / 2 \sqrt{|\boldsymbol{\beta}|^{2}+|\gamma|^{2}} .
$$

\section{Simulations}

In this section we will show the effects of the approximations to be small for a small field of view. We assume fixation and zero torsion $\left(\Omega_{\|}=-J \mathrm{~V}_{\|} / Z_{0}\right.$, $\Omega_{\perp}=0$ ) We take quadratic surface patches with a distance of $2.5 \mathrm{~m}$, slant zero, a curvedness of $5 \mathrm{~m}^{-1}$, direction of maximal curvature in the $x$ direction and a velocity of $1 \mathrm{~m} / \mathrm{s}$ in the $x$ direction. We take a square field of view of 6 by $6 \mathrm{deg}$. When we take a non-zero slant, we will always take zero tilt. We use this set of parameters in all simulations, unless otherwise noted. We took a grid of 5 by 5 in the image plane and calculated the velocity in each grid point. We fitted the velocity field with a polynomial up to second order using a linear least squares algorithm. From the fitted parameters we calculated the invariants and from there the estimates of the shape measures.

Because $\boldsymbol{\beta}$ enters in all our shape measures, it is important to show the effect of its non-curvature dependent terms. Using fixation and eq. 11, we find:

$$
\boldsymbol{\beta}=\left(Z_{X X}+Z_{Y Y}+2 / Z_{0}\right) \mathbf{V}_{\|}-2\left(V_{\perp} / Z_{0}\right) \nabla Z \text {. }
$$

There are two terms which we neglected. A zeroth order term $2 / Z_{0}$, which we assumed to be small relative to $Z_{X X}+Z_{Y Y}$, and a first order term $2\left(V_{\perp} / Z_{0}\right) \nabla Z$, which we assumed to be small relative to $\left(Z_{X X}+\right.$ $\left.Z_{Y Y}\right) \mathbf{V}_{\|}$. Note that the zeroth order term changes the length of $\beta$, not its direction. The first order term has a more complicated influence on $\boldsymbol{\beta}$, depending on the tilt. When $\nabla Z$ and $\mathbf{V}_{\|}$are parallel the first order term changes the length, not the direction of $\boldsymbol{\beta}$. When $\nabla Z$ and $\mathbf{V}_{\|}$are orthogonal the first order term not so much influences the length as the direction of $\boldsymbol{\beta}$.

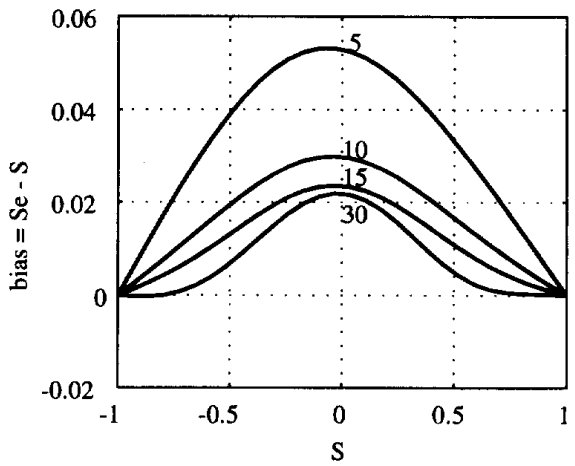

Figure 3 The bias as a function of shape index at the fixation point. The curves are for different values of the curvedness: $5,10,15$ and $30 \mathrm{~m}^{-1}$.

First, we show the effect of neglecting the zeroth order term in eq. 16 on the shape index $S_{e}$. In fig. 3 we plot the bias in shape index (difference between estimated shape index and shape index in the fixation point) for several values of the curvedness. Note that we would obtain the same curves for different values of $Z_{0}$ : keeping the curvedness constant at $5 \mathrm{~m}^{-1}$ and $Z_{0}$ equal to $15,7.5,5$ and $2.5 \mathrm{~m}$ results in the same curves. The bias is zero at the extremes of the shape index scale and increases towards the middle. One can show that the effect that the bias is zero for the spherical shapes, is caused by the vanishing of $\gamma$ at the extremes of the shape index scale. The bias is smaller for higher values of the curvedness.

Second, we show the effect of neglecting the first order term in eq. 16 and of neglecting the difference between $\lambda_{\max }, \lambda_{\min }$ and $\kappa_{\max }, \kappa_{\min }$ on the shape index $S_{e}$. In fig. 4 we plot the bias in shape index for different values of the slant and of the sign of $V_{\perp}$. It should be noted that the bias is also caused by the zeroth order term. The curve for zero slant is the same as the upper curve in the previous figure. We see that when $V_{\perp}>0$ i.e. when the observer is moving towards the surface, the bias is smaller than when $V_{\perp}<0$. This is the case because the first order term has a 


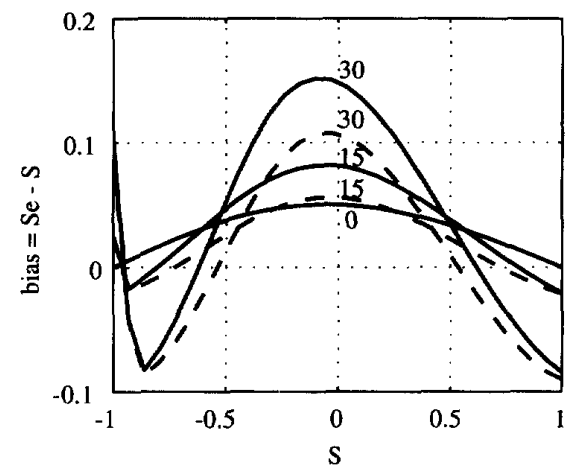

Figure 4 The bias in shape index as a function of shape index at the fixation point. The different curves are for different combinations of the slant and $V_{\perp}$. The two solid curves labeled 30 and 15 are for slant 30 and $15 \mathrm{deg}$ with $V_{\perp}=1$, the remaining solid curve is for slant zero $\left(V_{\perp}\right.$ does not matter). The dashed curves are for slant 30 and $15 \mathrm{deg}$ with $V_{\perp}=-1$. The other parameters are: curvedness 5 $\mathrm{m}^{-1}$, tilt zero, distance $2.5 \mathrm{~m}$ and $\mathrm{V}_{\|}=(1,0) \mathrm{m} / \mathrm{s}$.

different sign when $V_{\perp}>0$. In the figure we have plotted the best and worst case situations: when $\nabla Z$ and $\mathbf{V}_{\|}$are not parallel we find intermediate values of the bias. The discontinuity in the bias around shape index -0.9 is caused by a change of the sign of $\gamma$.

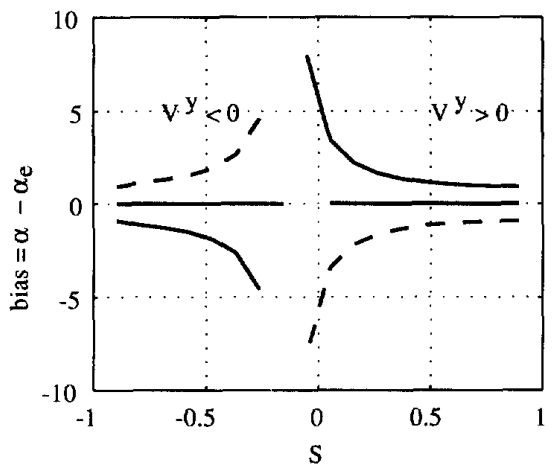

Figure 5 The bias in principal direction relative to the $x$ axis in $\operatorname{deg}$ as a function of shape index at the fixation point. The middle curve is for slant zero, the dashed discontinuous curve for slant $30 \mathrm{deg}$ and $\mathbf{V}=(0,-1,1)$ $\mathrm{m} / \mathrm{s}$ and the solid discontinuous curve for slant $30 \mathrm{deg}$ and $\mathbf{V}=(0,1,1) \mathbf{m} / \mathbf{s}$. The other parameters are: curvedness 5 $\mathrm{m}^{-1}$, tilt zero and distance $2.5 \mathrm{~m}$.

In fig. 5 we plot the bias in the principal direction (difference between $\alpha_{e}$ and $\alpha_{0}$ in the fixation point) for different values of the slant and velocity in the $y$ direction. We showed that $\alpha_{e}$ can be calculated from the angle between $\boldsymbol{\beta}$ and $\boldsymbol{\gamma}$. When either of these becomes very small the angle cannot be determined accurately. Therefore, we did not calculate $\alpha_{e}$ when the smaller of $\beta$ and $\gamma$ was less than $10 \%$ in length than the larger of the two. This happens around $S=$ $\pm 1,0$ which is why the curves are discontinuous at these values. For $S= \pm 1$ this is not a problem as $\alpha_{0}$ is not defined. In discussing the effects of neglecting the zeroth and first order term we already noted that only the first order term can change the direction of $\boldsymbol{\beta}$. We have chosen $\nabla Z$ and $\mathbf{v}_{\|}$orthogonal (the worst case) and still the difference does not exceed $8 \mathrm{deg}$.

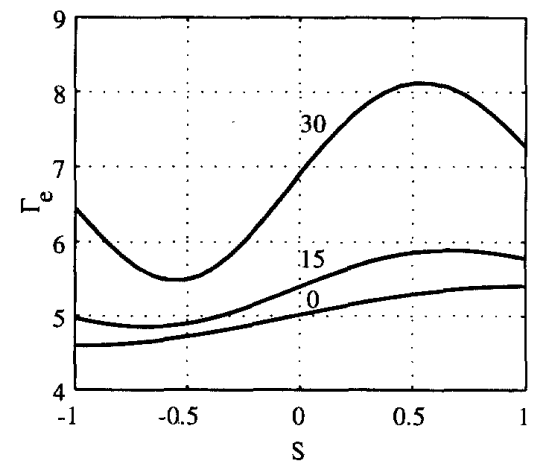

Figure 6 The velocity scaled curvature $\Gamma_{e}$ as a function of the shape index at the fixation point. The correct value is $5 \mathrm{~s}^{-1}$. The different curves are for different values of the slant: 0,15 and $30 \mathrm{deg}$. The other parameters are: curvedness $5 \mathrm{~m}^{-1}$, tilt zero, distance $2.5 \mathrm{~m}$ and $\mathrm{V}=(1,0,0) \mathrm{m} / \mathrm{s}$.

In fig. 6 we plot the velocity scaled curvedness $\Gamma_{e}$ for different values of the slant. The figure shows $\Gamma_{e}$ to be quite sensitive for slant. For higher values of the slant $\Gamma_{e}$ leads to a considerable overestimation of the true value. Further $\Gamma_{e}$ increases with $S$ for slant zero. One can show this to be caused by the zeroth order term in $\boldsymbol{\beta}$.

\section{Discussion}

We obtained an approximation to the absolute value of the shape index, to the curvedness and to the principal direction directly from the velocity field generated by a curved patch moving rigidly relative to a planar camera. Our approach is close to Koenderink and van Doorn's [3]. We improved on their paper by introducing the remaining second order invariant: the double deformation. We showed this invariant to be proportional to the difference of the maximal and minimal curvature. Our approach is different from [5]. First, Koenderink and van Doorn employed parallel projection whereas we use perspective projection. Second, their approach results in the projected indica- 
trix of Dupin, scaled by the slant and the 3D velocity, whereas some of our shape measures (shape index and principal direction) are relatively independent of both.
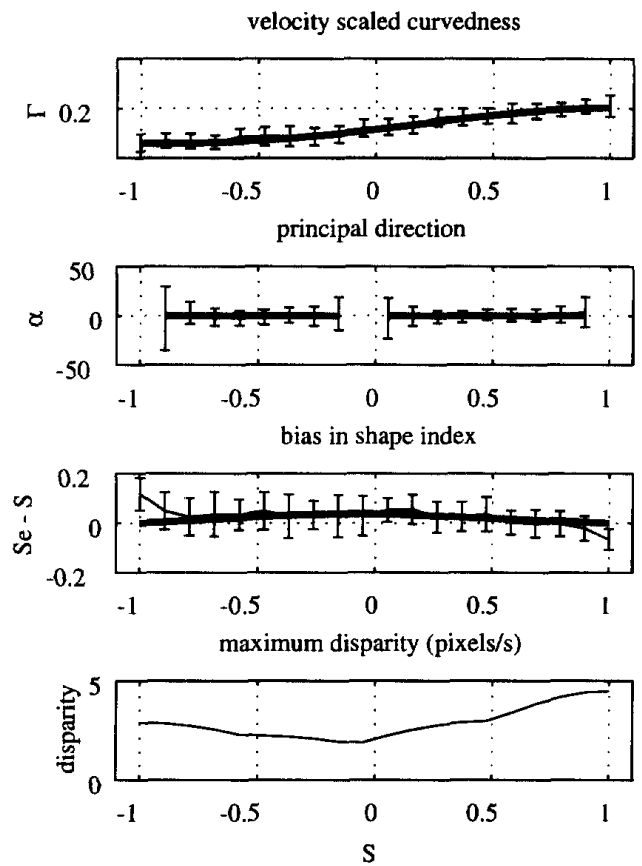

Figure 7 Top: velocity scaled curvedness $\Gamma_{e}$ as a function of the shape index at the fixation point. The correct value is $0.16 \mathrm{~s}^{-1}$. The thick line denotes the noiseless $\Gamma_{e}$, the thin line the mean of 30 simulations with additive noise. The errorbars denote standard deviation. Middle: same for principal directions and shape index. Lower: the maximal disparity on the camera. The other parameters are: curvedness $10 \mathrm{~m}^{-1}$, slant zero, distance $2 \mathrm{~m}$ and $\mathbf{V}=(0.016,0,0) \mathrm{m} / \mathrm{s}$.

We employ the velocity field as input to our calculations i.e. we assume a small disparity between two views of a smooth rigid curved surface patch. The discrete algorithms of structure from motion do not assume small disparity and smoothness [9]. For several reasons it is hard to compare our results directly to the results of this study. First, the discrete algorithms start with the calculation of the velocity and rotation of the camera and given these calculate the $3 \mathrm{D}$ position of the points. They do not calculate an explicit structure of the environment, although one could do this in an extra step by fitting a surface to the 3D points. This contrasts with our approach where we directly calculate the structure of the environment. Second, the discrete algorithms employ a two step approach: first, they use a linear algorithm to estimate the velocity and rotation of the camera. Because this estimate is statistically biased they use it as starting point for an iteration, which minimizes the discrepancy between fitted and observed image points. In contrast, our approach is linear. Third, the discrete algorithms are exact whereas ours is approximate. Notwithstanding these differences there is an interesting point in [9, Fig. 16)], where they did simulations for small disparity. They found the statistical bias in the estimate of the 3D velocity to be large: for 25 point correspondences and a maximal disparity of 4 pixels on a camera of 512 by 512 pixels they found the bias to be $50 \%$. We ran some simulations using a 10 deg field of view with gaussian white noise of standard deviation $0.01 \mathrm{deg} / \mathrm{s}$ added to the velocity field. This noise strength is comparable to that of [9]. The results (see fig. 7) show that our algorithm is quite robust. Only for the principal direction does the error become large which is caused by either $\boldsymbol{\beta}$ or $\boldsymbol{\gamma}$ becoming small.

This work was supported by the Foundation for Biophysics and by MUCOM (ESPRIT BRA 6615).

\section{References}

[1] T.M.H. Dijkstra, P.R. Snoeren and C.C.A.M. Gielen, "Extraction of 3D shape from optic flow: a geometric approach," to appear in J. Opt. Soc. Am. A 1994.

[2] K. Kanatani, Group-theoretical methods in image understanding, Springer, Berlin, 1990.

[3] J.J. Koenderink and A.J. van Doorn, "Invariant properties of the motion parallax field due to the movement of rigid bodies relative to an observer," Optica Acta, 22, 773-791, 1975.

[4] J.J. Koenderink and A.J. van Doorn, "Affine structure from motion," J. Opt. Soc. Am. A, 8, 377-385, 1991.

[5] J.J. Koenderink and A.J. van Doorn, "Second-order optic flow," J. Opt. Soc. Am. A, 9, 530-538, 1992.

[6] J.J. Koenderink and A.J, van Doorn, "Surface shape and curvature scales," Image and Vision Comp., 10, 557-565, 1992.

[7] B. O'Neill, Elementary differential geometry, Academic Press, New York, 1966.

[8] A.M. Waxman, B. Kamgar-Parsi and M. Subbarao, "Closed-form solutions to image flow equations for 3D structure and motion," Int. J. Comp. Vision, 1, 239-258, 1987.

[9] J. Weng, N. Ahuja and T.S. Huang, "Optimal motion and structure estimation," IEEE Trans. Pattern Anal. Machine Intell., 15, 864-884, 1993. 